\title{
Relato de experiência de Fisioterapeutas na atenção básica com mulheres no período de menopausa e climatério
}

\author{
Physiotherapists experience report on basic attention with women in the menopause and \\ climacteric period
}

Relato de experiencia de Fisioterapeutas en la atención básica con mujeres en el período de menopausia y climatério

José Felipe Costa da Silva ${ }^{1 *}$, Edson Mendes Marques ${ }^{2}$, Denise Rodrigues da Silva ${ }^{1}$, Joyce Raquel Cândido de Medeiros ${ }^{1}$, Rayssa Silva do Nascimento ${ }^{1}$, Juliana Simonelly Felix dos Santos ${ }^{1}$, Alisson Douglas da Nóbrega Correia ${ }^{1}$, Rita de Cássia Souza da Silva ${ }^{1}$, Risonety Maria dos Santos ${ }^{1}$, Jaine Maria de Pontes Oliveira ${ }^{1}$, Diasnara Eunice de Brito Cruz $^{3}$, Franciele Santos de Oliveira ${ }^{1}$.

\section{RESUMO}

Objetivo: O objetivo do presente trabalho é relatar uma experiência de abordagem da Fisioterapia na atenção básica com mulheres no período de menopausa e climatério. Métodos: As atividades foram realizadas por estudantes de fisioterapia da Faculdade de Ciências da Saúde do Trairi (FACISA/UFRN) com mulheres no período da menopausa e climatério, na cidade de Santa Cruz-RN entre os meses de agosto a setembro de 2014. Os encontros aconteciam uma vez por semana e duravam 01h30min. Relato de experiencia: Os encontros eram divididos em dois momentos: o primeiro com abordagens com educação em saúde com temas de interesse da mulher como: câncer de mama, câncer de colo de útero, incontinência urinária e importância de exercícios físicos. O segundo momento compreendeu: exercícios físicos aeróbicos, proprioceptivos e abordagem de treino para o assoalho pélvico. Conclusão: Os resultados mostram que o fisioterapeuta desempenha um papel importante na promoção e prevenção da saúde de mulheres nessa fase da vida.

Palavras-chave: Saúde da mulher, fisioterapia, Atenção Primária à Saúde.

\begin{abstract}
Objective: The aim of the present study is to report an experience of approaching physical therapy in basic care with women in the menopause and climacteric period. Methods: The activities were carried out by physiotherapy students from the Faculty of Health Sciences of Trairi (FACISA / UFRN) with women during the menopause and climacteric period, in the city of Santa Cruz-RN between August and September 2014. The meetings took place once a week and lasted one hour. Experience report: The meetings were divided into two moments: the first with approaches to health education with topics of interest to women such as breast cancer, cervical cancer, urinary incontinence and the importance of physical exercises. The second moment comprised: aerobic physical exercises, proprioceptive and training approach to the pelvic floor. Conclusion: The results show that the physiotherapist plays an important role in promoting and preventing the health of women in this phase of life.
\end{abstract}

Key words: Women's health, physical therapy, Primary Health Care.

\footnotetext{
${ }^{1}$ Universidade Federal do Rio Grande do Norte (UFRN), Santa Cruz-RN. *E-mail: felipedoshalom@yahoo.com.br

${ }^{2}$ Empresa Brasileira de Serviços Hospitalares (EBSERH) Hospital Universitário Ana Bezerra (HUAB).

${ }^{3}$ Faculdade Estácio de Natal.
} 


\section{RESUMEN}

Objetivo: El objetivo del presente trabajo es relatar una experiencia de la Fisioterapia en la atención básica con mujeres en el período de menopausia y climaterio. Métodos: Las actividades fueron realizadas por estudiantes de fisioterapia de la Facultad de Ciencias de la Salud del Trairi (FACISA / UFRN) con mujeres en el período de la menopausia y climaterio, en la ciudad de Santa Cruz-RN entre los meses de agosto a septiembre de 2014. Los encuentros ocurrían una vez por semana y duraban 01h30min. Relato de experiencia: Los encuentros se dividían en dos momentos: el primero con educación en salud con temas como: cáncer de mama, cáncer de cuello de útero, incontinencia urinaria e importancia de ejercicios físicos. El segundo momento comprendió: ejercicios físicos aeróbicos, ejercicios proprioceptivos y para los músculos del piso pélvico. Conclusión: Los resultados muestran que el fisioterapeuta desempeña un papel importante en la promoción y prevención de la salud de las mujeres en esta fase de la vida.

Palabras clave: Salud de la mujer, fisioterapia, Atención Primaria a la Salud.

\section{INTRODUÇÃO}

O Fisioterapeuta é um profissional que atua em diversas áreas do conhecimento da saúde, dentre as quais está à saúde da mulher. E com isso está inserido nos diversos níveis de atenção à saúde, desde a primária (conhecida também como atenção básica) até os níveis de maior complexidade. Nos últimos anos houve um aumento no número de fisioterapeutas inseridos na atenção básica, transformando os paradigmas de saúde individualista de saúde definido nas políticas públicas de saúde (BAENA CP e SOARES MCF, 2011; SOUZA MC et al., 2013).

A atenção básica é caracterizada por um conjunto de ações individuais e coletivas que abrange promoção e proteção da saúde, prevenção de agravos, diagnóstico, tratamento, reabilitação, redução de danos e a manutenção da saúde. No Brasil, a atenção básica deve se orientar pelos princípios do Sistema Único de Saúde (SUS) que são universalidade, equidade, integralidade, controle social, hierarquização. E sua prática deve ser executada o mais próximo da vida das pessoas através das Estratégias Saúde da Família (ESF), que é considerado como porta principal de entrada na rede de atenção à saúde (BRAIL, 2006; SIMÃO E et al., 2007; ALVES SB, et al., 2012).

No século $X X$ a saúde da mulher foi incorporada às políticas nacionais de saúde, no entanto os focos eram apenas a gravidez e o parto. No ano de 2004 o Ministério da Saúde lançou uma nova edição da Política Nacional de Atenção Integral à Saúde da Mulher, que tem como objetivo promover melhorias nas condições de vida e saúde das mulheres, garantindo direitos ao acesso a meios e serviços de promoção, prevenção, cura e recuperação da saúde, além de ser uma das prioridades do pacto pela vida. Nesta perspectiva o pacto pela vida se consolida como o compromisso entre os gestores do SUS para as prioridades de assistência à saúde. Esse pacto pela saúde tem referência para duas áreas relacionadas à saúde da mulher: controle do câncer do colo do útero e da mama e redução da mortalidade infantil e materna, além de outros pactos relacionados à saúde dos brasileiros (BRASIL, 2004; CARNEIRO TSG, et al., 2014; MACHADO RR, et al., 2009).

Enquanto profissional de saúde, o fisioterapeuta está apto a realizar atividades de promoção, prevenção, cura e recuperação à saúde, considerando todos os níveis de cuidado, a singularidade do sujeito e do meio ao qual ele está inserido. Em uma revisão, Portes LH et al. (2011) relataram que atividades como educação em saúde, atividades grupais e atendimento domiciliar são largamente realizadas pelo fisioterapeuta na Atenção Básica (PORTES LH, et al.,2011).

O Fisioterapeuta atuando em diversos ciclos da vida tendo grande importância em mulheres na menopausa e climatério, estudos demonstram que a atuação envolve educação em saúde para prevenção de agravos e adoecimento como câncer de mama, colo de útero e síndromes metabólicas, a treinamento específicos para prevenção e tratamento de Incontinência Urinária (IU) e ou fecal, distúrbios sexuais, posturais melhorando a qualidade de vida (OLIVEIRAAHFV et a., 2018; ALENCAR AB et al., 2018). 
A menopausa tardia é fator de risco associado ao câncer de mama. A mortalidade por câncer de mama no Nordeste está crescente, apesar de todas as idades serem acometidas, o envelhecimento é um fator de risco que se torna uma preocupação para mulher. Assim para essa população se torna necessário atividades que ensinem formas de prevenção e diagnóstico precoce promovendo a redução da mortalidade (BARBOSA IR, et al., 2015; SILVA RM, et al., 2015). A idade, fatores hereditários, menopausa, obesidade, constipação, e tabagismo são grandes fatores de risco para problemas como a IU, afetando mulheres de várias idades, o que reduz a autoestima, interfere na vida sexual e restringi atividades sociais (HIGA R, et al., 2008; HONÓRIO MO e SANTOS SMA, 2009).

A atividade física regular pelo menos três vezes por semana com intensidade moderada e de baixo impacto, traz melhorias na saúde física, aumenta o equilíbrio prevenindo agravos à saúde e melhorando a qualidade de vida. (BOTOGOSKI SR, et al, 2009; SOUZA MC, et al., 2015). Tendo em vista a importante atuação do fisioterapeuta na atenção básica principalmente através Núcleo Ampliado de Saúde da Família e Atenção Básica (NASF-AB), com foco relacionado à promoção da saúde e prevenção de agravos.

O objetivo do presente trabalho é relatar uma experiência da Fisioterapia na Atenção Básica com mulheres no período de menopausa e climatério.

\section{MÉTODOS}

Trata-se do relato de experiência, desenvolvida por acadêmicos do curso de graduação em Fisioterapia da Faculdade de Ciências da Saúde do Trairi- Universidade Federal do Rio Grande do Norte- FACISA/UFRN no munícipio de Santa Cruz/RN. As atividades foram realizadas em cumprimento à carga horária da disciplina de atenção fisioterapêutica na saúde da mulher na atenção básica no período de 19 de agosto a 18 de setembro de 2014.

Os encontros aconteciam uma vez por semana nas terças-feiras no período vespertino com duração de $1 \mathrm{~h}$ e 30min, o local utilizado para ação era a quadra do Instituto Federal do Rio Grande do Norte em Santa Cruz/RN, o público alvo foram mulheres no período de menopausa e climatério que frequentavam um grupo de atividade física no presente local.

Os encontros eram divididos em dois momentos. No primeiro era realizada a educação em saúde com exposição de imagens, roda de conversas e prática dos assuntos exposto durando 15 a $20 \mathrm{~min}$. O segundo momento com duração média de 30 a 40 min, era focado nas atividades físicas com aquecimento, exercícios aeróbicos, treino proprioceptivo e fortalecimento de Musculatura do Assoalho Pélvico (MAP) e desaquecimento.

\section{RELATO DA EXPERIÊNCIA E DISCUSSÃO}

A educação em saúde se baseou em estudos de Ribeiro SB e Lima KRP (2015) que demonstra o Fisioterapeuta como agente educador promovendo cuidado.

O câncer do colo do útero e de mama foram abordados através de rodas de conversas debatendo os fatores de risco, a importância do exame ginecológico preventivo e autoexame. Em seguida, as mulheres eram incentivadas a reproduzirem seu aprendizado através do autoexame. Esses temas são importantes pois estudos demonstram que a maioria das mulheres desconhecem os fatores que desencadeiam o câncer de mama e as principais formas de prevenção (RIBEIRO SB e LIMA KRP, 2015; SILVA RM, et al., 2015).

O segundo momento foi ofertado atividades físicas com alongamento global dos principais grupos musculares, atividades de aquecimento como caminhada rápida e/ou dança. O Treino aeróbico era o primeiro a ser realizado com utilização de músicas incentivadoras. A propriocepção era treinada em superfícies instáveis como na cama elástica onde eram feitos agachamentos com ou sem bastão e ficando alguns segundos em posição unipodal. 
Estudo feito em mulheres concluem que essas atividades diminuem os riscos de quedas (CALLEGARI B, et al., 2010; SANTOS AA, et al., 2008).

Segundo relatos das participantes algumas possuíam IU. Por essa razão, os exercícios eram focados no fortalecimento da MAP, como forma de prevenir ou tratar a incontinência urinária.

A prevenção e o tratamento buscam a realização de exercícios que aumentem a força da musculatura pélvica, conhecimento e controle muscular reforçando assim o mecanismo de continência e a coordenação reflexa durante o esforço (SANTOS AA, et al., 2008; OLIVEIRA KAC, et al., 2007).

Experiencias baseadas em (Kaetsner 2016) foram inseridas e segundo o autor é eficaz para melhorar o nível de conhecimento da prevenção da IU, atividades exemplificadas com figuras da formação do assoalho pélvico e seus componentes, a importância dessas estruturas musculares na prevenção da perda da urina e ou fezes e função sexual, e como é possível prevenir esses problemas através de exercícios específicos para o fortalecimento do MAP que foram ensinados em todos os encontros (KAESTNER KT, et al., 2016).

Os exercícios para os MAP segundo a literatura mostram grandes resultados na prevenção das IU além de melhorias na autoestima das mulheres. Eles foram realizados na posição de pé, sentado ou deitado. Agachamento e ponte eram os mais usados e associados com a contração isométrica dos MAP durante 15 segundos, juntamente com contração e relaxamento de uma forma dinâmica e rápida da musculatura pélvica até 45 vezes por minuto (MARQUES KSF e FREITAS PAC, 2005; ALVES FK, et al., 2016; VIANA R, et al., 2014).

\section{CONSIDERAÇÕES FINAIS}

A percepção dos profissionais envolvidos no presente relato de experiência indicou uma correlação positiva da Fisioterapia na Atenção Primária na saúde da mulher. Afirmando que o fisioterapeuta não está apenas qualificado para cuidar da reabilitação dos pacientes em ambulatório, mas está apto a desempenhar um papel importante na educação em saúde dos membros da comunidade sobre assuntos pertinentes a saúde integral da população. E que as ações realizadas na área de atenção à saúde da mulher; além de prevenir complicações de saúde em mulheres na menopausa e climatério, inclui a atividade física como fator preponderante na promoção à saúde. A integralidade do cuidado deve, portanto, considerar a complexidade do conceito ampliado em saúde e não somente se restringir exclusivamente a ações de cura e reabilitação ao qual, muitas vezes, estamos equivocadamente acostumados a realizar nos serviços de saúde.

\section{REFERÊNCIAS}

1. ALENCAR AB, et al. Avaliação do nível de atividade física e dos sintomas climatérios em mulheres em menopausa acometidas por síndrome coronariana aguda. Cad Grad Ciên. Bio. E Saú -UNIT-ALA. 2017; 4(2): $227-238$.

2. ALVES FK, et al. Inserção de um programa de treinamento dos músculos do assoalho pélvico na Atenção Básica à Saúde para mulheres na pós-menopausa. Fisio. Bra. 2016;17(2):131-139.

3. ALVES SB, et al. Manejo de resíduos gerados na assistência domiciliar pela Estratégia de Saúde da Família. Rev. bras. enferm. 2012;65(1):128-134.

4. BAENA CP, SOARES MCF. Fisioterapia e integralidade: novos conceitos, novas práticas: estamos prontos? Rev Fisio Bras. 2011; 12(2):133-138.

5. BARBOSA IR, et al. Mortalidade por câncer de mama nos estados do nordeste do Brasil: tendências atuais e projeções até 2030. Revista Ciência Plural. 2015;1(1):4-14.

6. BOTOGOSKI SR, et al. Os Benefícios do exercício físico para mulheres após a menopausa. Arq Med Hosp Fac Cienc Med Santa Casa São Paulo. 2009;54(1):18-23.

7. BRASIL. Ministério da Saúde (MS). Política nacional de atenção integral à saúde da mulher: princípios e diretrizes. Brasília: MS, 2004.

8. BRASIL. Ministério da Saúde. Secretaria de atenção à saúde. departamento de atenção básica. Política nacional de atenção básica. Ministério da Saúde, 2006.

9. CALLEGARI $\mathrm{B}$, et al. Atividade eletromiográfica durante exercícios de propriocepção de tornozelo em apoio unipodal. Fisioter. Pesqui. 2010;17(4):312-6. 
10. CARNEIRO TSG, et al. O pacto pela saúde na prática cotidiana da atenção primária à saúde. Saúde em debate. 2014 set;38(102):429-39.

11. HIGA R, et al. Fatores de risco para incontinência urinária na mulher:[revisão]. Rev. Esc. Enferm. USP. 2008;42(1):187-192.

12. HONÓRIO MO, SANTOS SMA. Incontinência urinária e envelhecimento: impacto no cotidiano e na qualidade de vida. Rev. Bras. Enferm. 2009;62(1):51-6.

13. KAESTNER KT, et al. Eficácia da dinâmica de grupo em educação em saúde em mulheres com incontinência urinária. Arq. Ciênc. Saúde. 2016 abr-jul;23(2):54-59.

14. MACHADO RR, et al. Entendendo o pacto pela saúde na gestão do SUS e refletindo sua implementação. Rev. Eletr. Enf. [Internet]. 2009;11(1):181-7.

15. MARQUES KSF, FREITAS PAC. A cinesioterapia como tratamento da incontinência urinária na unidade básica de saúde. Fisioter. Mov.2005;18(4):63-67.

16. OLIVEIRA KAC, et al. Técnicas fisioterapêuticas no tratamento e prevenção da incontinência urinária de esforço na mulher. Rev. Eletr. F@pci. 2007;1(1):31-40.

17. OLIVEIRA AHFV et al. Contribuições da fisioterapia na incontinência urinária no climatério. Rev. Ciên. Méd. 2018;26(3):127-133.

18. PORTES LH, et al. Atuação do fisioterapeuta na atenção básica à saúde: uma revisão da literatura brasileira. Rev. APS. 2011;14(1):111-119.

19. RIBEIRO SB, LIMA KRP. A Importância do fisioterapeuta como agente educador de saúde na unidade básica de saúde da família. Rev Bras Saúd Fun. 2015;2(2):05-14.

20. SANTOS AA, et al. Efeito do treinamento proprioceptivo em mulheres diabéticas. Rev Bras Fisioter. 2008;12(3):183-7.

21. SILVA RM, et al. Educação em saúde para prevenção do câncer de mama no município de Piripiri-Pi: Atuação Do Pet-Saúde. R. Epidemiol. Control. Infec. 2015;5(4):203-205.

22. SIMÃO E et al. Atenção básica no Brasil (1980-2006): Alguns destaques. Rev Rene. 2007;8(2):50-9.

23. SOUZA MC, et al. Fisioterapia e núcleo de apoio à saúde da família: conhecimento, ferramentas e desafios. Mundo Saúde. 2013;37(2):176-18

24. SOUZA MC, et al. Fisioterapia, acesso e necessidades de saúde: limites e possibilidades na atenção básica. Rev. Pesq. Fisio. 2015;5(2):125-133.

25. VIANA R, et al. Fisioterapia na autoestima de mulheres com incontinência urinária: estudo longitudinal. Psicol Saú. \& Doen. 2014;15(1):169-178. 